\title{
Optical Densities Functions of Lens and Macular Pigment Estimated from the Color Matching Functions
}

\author{
Yoshinobu Nayatani*, Kotaro Takahama** and Hiroaki Sobagaki**
}

\begin{abstract}
The eye lens and the macular pigment have been treated to be transparent for the long wavelengths. On the other hand, the observer variations of the color matching functions (CMF's) suggests that the lens and the macular pigment have unneglisible densities in the long wavelengths. In the present paper, improved values for the optical densities are proposed on the basis of the observer variation of CMF's. First, the principal component analysis is applied to the red CMF's of Stiles' 20 observers. The first eigen vector and the second eigen vector correspond to the spectral density functions of lens and macular pigment, respectively. Improved values of densities of lens and macular pigment are defined by connecting the published data of densities with the corresponding eigen vectors. Next, CMF's of Stiles' 20 observers are estimated by using the improved values of densities. It is confirmed that these estimated CMF's fit well the measured CMF's for the X component of CMF compared with the estimated CMF's from the published data though there is not an appreciable improvement for the $\mathrm{Y}$ and the $\mathrm{Z}$ component. Finally, the method of singular value decomposition is applied to the estimated CMF's from the improved densities. Deviate functions derived agree well with the deviate functions based on the measured CMF's.
\end{abstract}

\section{Introduction}

The authors showed in a previous report ${ }^{1 \text { ) }}$ that as a result of the analysis of the blue color

$\uparrow$ This paper is translated from Vol. 72 No. 10 of the Journal of IEIJ.

* Osaka Electro-Communication University

** Electrotechnical Laboratory, Osaka Life Electronics Research Center matching functions (CMF's) $\bar{b}(\lambda)$ of Stiles' 20 observers $^{2)}$ the individual variation of the blue CMF's could be explained by the individual variation of the spectral optical density $D_{1}(\lambda)$ and $D_{2}(\lambda)$ of eye lens and mascular pigment, respectively, from which the CMF's $\bar{r}(\lambda), \bar{g}(\lambda)$, $\bar{b}(\lambda)$ could be estimated sufficiently. In the previous report, the blue CMF $\bar{b}(\lambda)$ was used for analysis because the blue color lies in the wavelength band in which $D_{1}(\lambda)$ and $D_{2}(\lambda)$ change remarkably and they have sensitivity.

By the way, when the actual measured CMF's of each observer were compared with the estimated CMF's from $D_{1}(\lambda)$ and $D_{2}(\lambda)$ in detail, a remarkable difference was noticed for the red CMF $\bar{r}(\lambda)$ and $\bar{x}(\lambda)$ in the long wavelength region. It was then estimated that this difference was due to the fact that the published data 3 ) of $D_{1}(\lambda)$ and $D_{2}(\lambda)$ was almost equal to zero or flat in the band of $\lambda \geqq 550 \mathrm{~nm}$.

During the process of these studies, the authers supposed that if CMF's could be estimated from $D_{1}(\lambda)$ and $D_{2}(\lambda)$, improved values of $D_{1}(\lambda)$ and $D_{2}(\lambda)$ would be estimated from CMF's conversely. The present report will describe the procedure for estimating the improved values of $D_{1}(\lambda)$ and $D_{2}(\lambda)$ on the basis of this idea and the possibility of obtaining better estimated values of the CMF's of Stiles' 20 observers by using the improved estimated values of $D_{1}(\lambda)$ and $D_{2}(\lambda)$.

\section{Analysis of Red CMF $\bar{r}(\lambda)$}

First, suppose the ratios of CMF's $\bar{r}_{p}(\lambda)$, $\overline{\mathrm{g}}_{p}(\lambda), \bar{b}_{p} \mathrm{p}(\lambda), \mathrm{p}=1,2 . \ldots ., 20$ of Stiles' 20 observers to the observers' mean CMF's $\overline{\bar{r}}(\lambda)$, $\overline{\bar{g}}(\lambda), \overline{\bar{b}}(\lambda)$. The ratios $\overline{\mathrm{r}}_{\mathrm{p}}(\lambda) / \overline{\overline{\mathrm{r}}}(\lambda), \overline{\mathrm{g}}_{\mathrm{p}}(\lambda) / \overline{\overline{\mathrm{g}}}(\lambda)$, $\bar{b}_{p}(\lambda) / \overline{\bar{b}}(\lambda)$, are the relative ratios of the 
spectral transmittance in the optical system from eye surface to retina cell for an observer $p$ to the spectral transmittance for the average observer. Furthermore, the same observer has the same eyeball optical system irrespective of the type of CMF's so that the ratios $\overline{\mathrm{r}}_{\mathrm{p}}(\lambda) / \mathrm{r}(\lambda)$, $\overline{\mathrm{g}}_{\mathrm{p}}(\lambda) / \overline{\overline{\mathrm{g}}}(\lambda), \overline{\mathrm{b}}_{\mathrm{p}}(\lambda) / \overline{\overline{\mathrm{b}}}(\lambda)$ give the same estimated value for the relative spectral transmittance.

The purpose of the present report is to derive improved estimated values of the optical densities of eye lens and macular pigment in the band including long wavelengths, as stated in Introduction. Therefore, it was decided to analyse the red CMF's $\overline{\mathrm{r}}_{\mathrm{p}}(\lambda)$ because the red $\mathrm{CMF}$ has rather high values not equal to zero in the whole wavelength band of 390 to $720 \mathrm{~nm}$. Further, in order to relate CMF's to spectral optical density of eye's optical system, the following logalithmic functions were analyzed:

$$
\mathrm{u}_{\mathrm{p}}(\lambda)=\log \left[\overline{\mathrm{r}}_{\mathrm{p}}(\lambda) / \overline{\overline{\mathrm{r}}}(\lambda)\right], \quad \ldots \ldots
$$

where $p=1,2, \ldots . ., 20$ and $\lambda=$ each of 34 wavelengths (10 nm interval) in 390 to $720 \mathrm{~nm}$.

A principal component analysis was applied to inter-wavelength variance-covariance matrix $\left(V_{i j}\right)$ defined by

$V_{i j}=\frac{1}{19} \sum_{p=1}^{20}\left[u_{p}\left(\lambda_{i}\right)-\bar{u}\left(\lambda_{i}\right)\right]\left[u_{p}\left(\lambda_{j}\right)-\bar{u}\left(\lambda_{j}\right)\right]$,

where

$$
\overline{\mathrm{u}}\left(\lambda_{\mathrm{i}}\right)=\frac{1}{20} \sum_{\mathrm{p}=1}^{20} \mathrm{U}_{\mathrm{p}}\left(\lambda_{\mathrm{i}}\right)
$$

contribution factors of the 1st, the 2nd and the 3rd principal component were 86.7, 8.0 and $1.9 \%$, respectively. From the reason that the cumulative contribution factor of the two components are sufficiently high such as $94.7 \%$ and that the 3rd eigen value is low and the corresponding eigen vector is irregular in shape, only the 1st and the 2nd component were selected in the same way as the previous report. Table 1 shows the values of the 1 st and the 2 nd eigen vector. Table 2 shows the 1 st and the 2 nd principal component scores for Stiles' 20 observers.
Table 1 Value of the 1st and the 2nd eigen vector $L_{1}$ and $L_{2}$ derived from red CMF's

\begin{tabular}{cccccc}
\hline $\begin{array}{c}\text { Wave- } \\
\text { length }\end{array}$ & $\mathrm{L}_{1}$ & $\mathrm{~L}_{2}$ & $\begin{array}{c}\text { Wave- } \\
\text { length }\end{array}$ & $\mathrm{L}_{1}$ & $\mathrm{~L}_{2}$ \\
\hline 390 & 0.701 & -0.355 & 560 & 0.030 & 0.092 \\
400 & 0.475 & -0.221 & 570 & 0.033 & 0.091 \\
410 & 0.328 & 0.059 & 580 & 0.029 & 0.072 \\
420 & 0.230 & 0.192 & 590 & 0.026 & 0.078 \\
430 & 0.195 & 0.295 & 600 & 0.024 & 0.068 \\
440 & 0.120 & 0.491 & 610 & 0.019 & 0.049 \\
450 & 0.143 & 0.384 & 620 & 0.013 & 0.029 \\
460 & 0.119 & 0.314 & 630 & 0.007 & 0.003 \\
470 & 0.091 & 0.183 & 640 & -0.002 & -0.002 \\
480 & 0.076 & 0.144 & 650 & -0.002 & -0.004 \\
490 & 0.067 & 0.128 & 660 & -0.005 & -0.016 \\
500 & 0.053 & 0.124 & 670 & -0.009 & -0.029 \\
510 & 0.050 & 0.145 & 680 & -0.008 & -0.039 \\
520 & 0.040 & 0.183 & 690 & -0.005 & -0.051 \\
530 & 0.036 & 0.090 & 700 & -0.005 & -0.055 \\
540 & 0.042 & 0.072 & 710 & -0.004 & -0.052 \\
550 & 0.035 & 0.092 & 720 & -0.004 & -0.047 \\
\hline
\end{tabular}

Table 2 Value of the 1st and the 2nd principal component scores $C_{p}{ }^{(1)}$ and $\mathrm{Cp}^{(2)}$ for each observer derived from red CMF's

\begin{tabular}{lcrr}
\hline Observer & Age & \multicolumn{1}{c}{$\mathrm{Cp}^{(1)}$} & \multicolumn{1}{c}{$\mathrm{Cp}^{(2)}$} \\
\hline WSS & 54 & -0.980 & 0.190 \\
JMB & 30 & 0.276 & -0.196 \\
EMK & 27 & 0.579 & -0.281 \\
GEVL & 49 & -0.510 & -0.359 \\
MCB & 27 & 0.652 & 0.046 \\
DLT & 50 & -0.009 & -0.206 \\
EML & 34 & -0.718 & 0.141 \\
MHP & 44 & -0.214 & -0.118 \\
EA & 28 & 0.193 & -0.141 \\
RNW & 28 & -0.061 & 0.163 \\
MG & 22 & 0.675 & 0.202 \\
PSW & 31 & 0.720 & 0.084 \\
FJJC & 22 & 0.752 & 0.194 \\
JAR & 22 & 1.123 & 0.005 \\
W & 29 & -0.444 & 0.502 \\
JSP & 50 & -0.872 & 0.271 \\
WDW & 50 & 0.043 & -0.045 \\
JMB & 30 & 0.352 & -0.158 \\
GW & 34 & 0.265 & -0.031 \\
DBJ & 59 & -1.823 & -0.262 \\
\hline
\end{tabular}

In Figs. 1 and 2, the 1st and the 2nd eigen vector are shown with the mark $\bullet$. In these figures, the curves marked with the mark 0 show the published data ${ }^{3)}$ of optical densities of lens and macular pigment. In these cases, the scales of the eigen vectors are adjusted so as to be able 
to compare them with these published data; i.e. the 1st and the 2 nd eigen vector are multiplied by 2,359 and 1,250 respectively. These adjustment factors were selected such that the values of eigen vectors of lens and macular pigment conform to the published data in the wavelength band of $390 \sim 660 \mathrm{~nm}$ and $410 \sim 540 \mathrm{~nm}$, respectively, in the sense of least squares method.

From Fig. 1 and 2, the following conclusions were obtained.

(1) Concerning the lens spectral optical density, the conformity of the 1st eigen vector to the published data is very well.

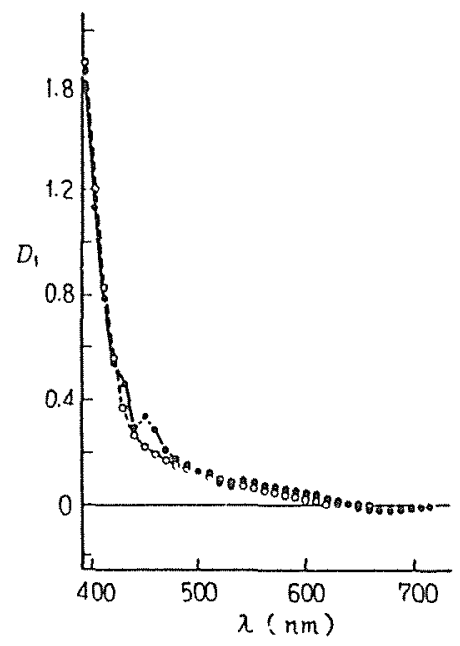

Fig. 1 Comparison between the 1st eigen vector and lens spectral optical density -... the 1st eigen vector

$\circ$... published data ${ }^{3)}$ of lens spectral optical density

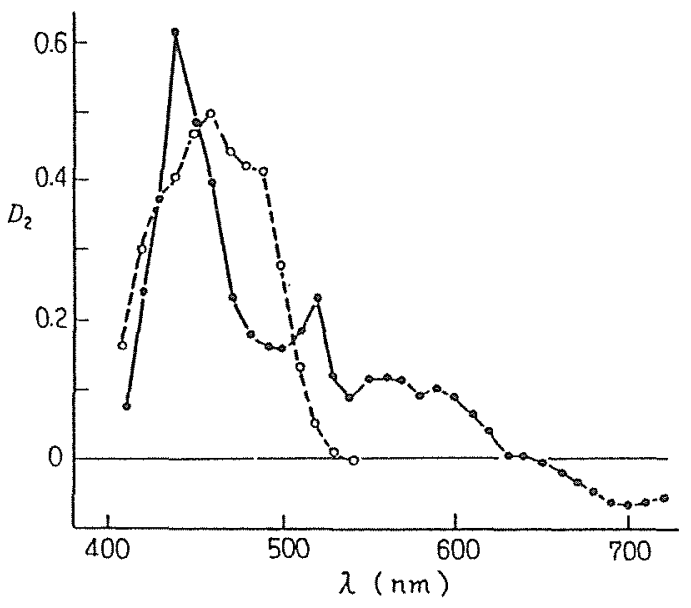

Fig. 2 Comparison between the 2nd eigen vector and macular pigment. spectral optical density

-... the 2nd eigen vector

-... published data ${ }^{3)}$ of macular pigment spectral optical density
(2) Concerning the macular pigement spectral optical density, the conformity is a little bad. It is caused by the lower contribution factor of the 2nd principal component. The main difference is that in the band below $450 \mathrm{~nm}$ the 2nd eigen vector is higher than the published data and in the band above $450 \mathrm{~nm}$ conversely lower.

(3) The values of macular pigment spectral optical density in the published data are zero and flat above $540 \mathrm{~nm}$ as shown in Fig. 2, while the eigen vector decline gently in the band from 500 to $700 \mathrm{~nm}$.

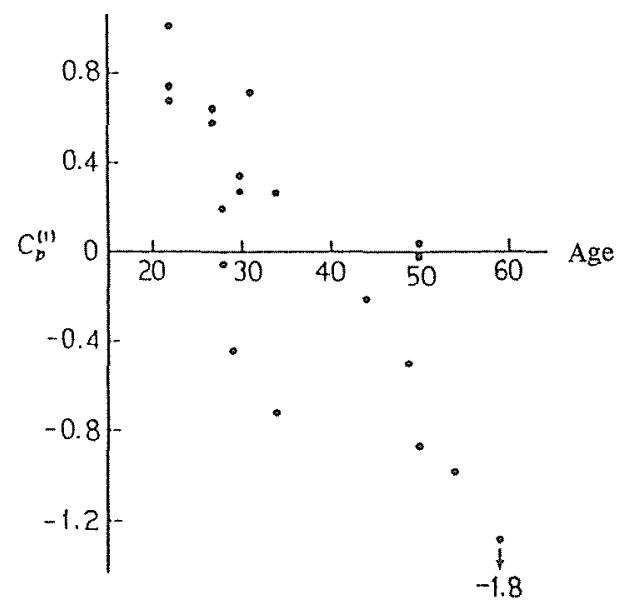

Fig. 3 Correlation between the 1st principal component point $C p^{(1)}$ and observer's age

Figure 3 shows a correlation between the 1st component score and the observer's age. It is obvious from the figure that the correlation between the age and the 1st principal component score is significant and it suggests an age effect of lens. On the other hand, no correlation was found between the 2nd principal component score corresponding to macular pigment and the observer's age. These results agree well with the known facts.3)4)

\section{Examination and Improved Estimation of Spectral Optical Densities of Lens and Macular Pigment}

In order to obtain improved estimated values for the published data of spectral optical densities of lens and macular pigment from the eigen vectors derived in the last paragraph, the rotation of eigen vector, the deviation of the 
published data from the estimated values for macular pigment optical density in the band of $400 \sim 500 \mathrm{~nm}$, and the variation of macular pigment optical density in the band of $500 \sim 700$ $\mathrm{nm}$ were studies. In addition, based on these results, improved estimated values of the spectral optical densities of lens and macular pigment were determined.

\subsection{Rotation of Eigen Vector}

In the principal component analysis method, an axis explaining correctly the variation of populational multivariate data mathematically is selected as the 1st principal component axis $L_{1}(\lambda)$ and an axis being orthogonal to $L_{1}(\lambda)$ and explaining the variation of the other data excluded from the effect of the 1st principal component is selected as the 2nd principal component axis $L_{2}(\lambda) . L_{1}(\lambda)$ and $L_{2}(\lambda)$ do not necessarily conform to the physical existance so that the values of coordiates $L_{1}(\lambda, \theta)$ and $L_{2}(\lambda, \theta)$ rotated from $L_{1}(\lambda)$ and $L_{2}(\lambda)$ by an arbitrary angle $\theta$ are also capable of equivalent alternatives for the physical existence.

$\left[\begin{array}{l}L_{1}(\lambda, \theta) \\ L_{2}(\lambda, \theta)\end{array}\right]=\left[\begin{array}{rr}\cos \theta & -\sin \theta \\ \sin \theta & \cos \theta\end{array}\right]\left[\begin{array}{l}L_{1}(\lambda) \\ L_{2}(\lambda)\end{array}\right] \ldots \ldots$

By rotating the axes of coordinates in the direction $\left(\theta=-5^{\circ},-10^{\circ},-15^{\circ},-20^{\circ}\right)$ in which direction the conformity to the spectral optical densities of lens and macular pigment may be improved, $L_{1}(\lambda, \theta)$ and $L_{2}(\lambda, \theta)$ were derived. However, an improvement was not almost found by the rotation.

Therefore, it was decided to use the curves marked with the mark $\bullet$ in Figs. 1 and 2, which were not rotated, as the estimated values of the spectral optical densities of lens and macular pigment.

It is supposed that the fact that the result of the principal component analysis method reflected directly the spectral optical densities of lens and macular pigment is based on the following reasons; that the correlation between the spectral optical densities of lens and macular pigment was low, that the contribution factor was excessively higher in the 1st principal component than in the 2nd principal component and that a relating physiological structure agreed very well to the principal component analysis model. For example, the correlation coefficient $r$ between spectral optical densities of lens and macular pigment is $r=0.033$ in the wavelength band of $400 \sim 570 \mathrm{~nm}$ so that the two vectors are almost orthogonal.

\subsection{Macular Pigment Spectral Optical Densi- ty in the Short Wavelength Band below $500 \mathrm{~nm}$}

In the short wavelength band below $500 \mathrm{~nm}$, as shown in Fig. 2, the peak of the density curve estimated from the CMF's is shifted towards a shorter wavelength compared with the density curve according to the published data.

In order to study this fact, the same principal component analysis was applied to the blue CMF's in the wavelength band of $390 \sim 550 \mathrm{~nm}$ as the one applied to the red CMF's, as described above. Based on this analysis, the values corresponding to the macuiar pigment spectral optical density were estimated from the 2nd principal eigen vectors. The results are shown in Fig. 4 together with the published data, the scale being adjusted in the same way as Fig. 2 .

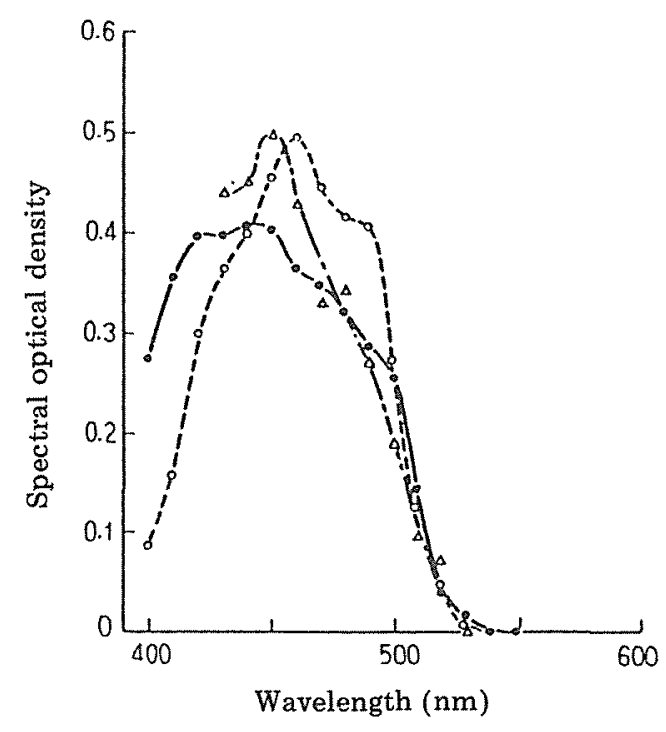

Fig. 4 Comparison of macular pigment spectral optical densities

-... The 2nd eigen vector obtained by the principal component enalysis of the CMF's in the short wavelength band

o... Published data of macular pigment spectral optical density

$\triangle$... Meassured value by Naylor ${ }^{5)}$ et al. 
In Fig. 4, the curve with the mark $\circ$ is the published data and the curve with the mark $\bullet$ is the estimated value from the result of the principal component analysis for blue CMF's. In this principal component analysis, the contribution factors of the 1st and the 2nd principal component are 85.1 and $11.2 \%$, respectively, which conform well to the result obtained in Paragraph 2.

The macular pigment spectral optical density (mark •) estimated from CMF's, as shown in Figs. 2 and 4, does not necessarily conform well to the published data due to the lower contribution factor of the 2nd principal component. Rather, in the wavelength band below $450 \mathrm{~nm}$, the estimated values of macular pigment spectral optical density are fairly higher than the published data.

In Fig. 4, the measured values by Naylor et al.5) are also shown together for reference. The published data in Figs. 2 and 4 are based on the Stiles'6) measurement result. Its result was obtained from the comparison of the threshold value in macular portion with the threshold value in the other portion exclusive of macular.

On the other hand, the values of Naylor et al. were measured by uisng the phenomenum called "Haidinger's brushes." Wyszecki-Stiles ${ }^{3)}$ disregard the measurement results of Naylor et al. because their accuracy is lower. However, if the above-mentioned estimation reuslts from CMF's are taken into consideration, it will not be necessary said that the measurement results of Naylor et al. are unsatisfactory.

\subsection{Macular Pigment Spectral Optical Densi- ty in the Long Wavelength Band above $500 \mathrm{~nm}$}

As already described, the published data of macular pigment spectral optical density are zero in the wavelength band above $500 \mathrm{~nm}$. On the other hand, the 2nd principal component vectors relating to $\log \left[\overline{\mathrm{r}}_{\mathrm{p}}(\lambda) / \overline{\overline{\mathrm{r}}}(\lambda)\right]$ decline gently over $500 \sim 700 \mathrm{~nm}$. As the values of spectral optical density are low in this wavelength band, the measurement may not be so easy. Even in the summary of a variety of past measurements of macular pigment spectral optical density by Wyszecki-Stiles, the measurement results in the wavelength band above 600 $\mathrm{nm}$ have not been reported.
In order to study the suggested tendency of spectral optical density in the long wavelength band, the red CMF's estimated from the published data of spectral optical density were compared with the red CMF's obtained in the same way from the estimated values of the spectral optical values shown in Figs. 1 and 2.

The estimation of the former was already made in the previous report. ${ }^{1)}$ The estimation of the latter will be described as follows. By the way, the lens spectral optical density show a good confirmity of the published data to the estimated values by the principal component analysis so that it is expected that the difference between the published data and the estimated value of macular pigment spectral optical density generates the deviation in the long wavelength band in the above-mentioned comparison.

Now, by using the result of the principal component analysis as described in Paragraph 2, $\mathrm{u}_{\mathrm{p}}$ $(\lambda)=\log \left[\bar{r}_{p}(\lambda) / \overline{\bar{r}}(\lambda)\right]$ is estimated by

$\left\langle\mathrm{u}_{\mathrm{p}}(\lambda)\right\rangle=\overline{\mathrm{u}}(\lambda)+\mathrm{C}_{\mathrm{p}}{ }^{(1)} \mathrm{L}_{1}(\lambda)+\mathrm{C}_{\mathrm{p}}{ }^{(2)} \mathrm{L}_{2}(\lambda)$,

where $\overline{\mathrm{u}}(\lambda)$ is given by Eq. (3) and the values of eigen vectors $L_{1}(\lambda), L_{2}(\lambda)$ and principal component scores $\mathrm{C}_{\mathrm{p}}{ }^{(1)}, \mathrm{C}_{\mathrm{p}}{ }^{(2)}$ are calculated in Paragraph 2. By using Eq. (5), the deviation from the mean CMF's is calculated by

$$
\begin{aligned}
\left\langle\Delta \overline{\mathrm{r}}_{\mathrm{p}}(\lambda)\right\rangle & =\left\langle\overline{\mathrm{r}}_{\mathrm{p}}(\lambda)-\overline{\overline{\mathrm{r}}}(\lambda)\right\rangle \\
& =\overline{\overline{\mathrm{r}}}(\lambda)\left[10^{\left\langle\mathrm{u}_{\mathrm{p}}(\lambda)\right\rangle}-1\right] \ldots
\end{aligned}
$$

The conformity to the actual measured CMF's deviations were better in the deviations estimated from Eq. (6) than in the deviations estimated by using $D_{1}(\lambda)$ and $D_{2}(\lambda)$, as described in the previous report. ${ }^{1)}$ As a result of study on total observers, an improvement of conformity was confirmed in 14 persons among 20 observers, 2 persons had the same conformity as the previous report and the conformity of residual 4 persons was worsened. From these results, it is suspected that it is reasonable to consider that the macular pigment spectral optical density has a declining characteristic in the long wavelength band above $500 \mathrm{~nm}$.

\subsection{Improved Estimation of Spectral Optical Densities of Lens and Macular Pigment}


On the basis of the above-mentioned study, the improved estimated values $\dot{D}_{1}(\lambda)$ and $\dot{D}_{2}(\lambda)$ of the spectral optical densities of lens and macular pigment are determined as follows.

(1) $\dot{\mathrm{D}}_{1}(\lambda)$ : In the band of $390 \sim 640 \mathrm{~nm}$ $D_{1}(\lambda)$ of the published data is used and in the band of $650 \sim 720 \mathrm{~nm}$ the value of $L_{1}(\lambda)$ shown in Fig. 1 is used.

(2) $\dot{\mathrm{D}}_{2}(\lambda)$ : In the band below $500 \mathrm{~nm}$ some information suggests the shift of the peak wavelength towards the short wavelength side. However, the estimated value from the principal component analysis result depends whether $\overline{\mathrm{r}} \mathrm{p}$ $(\lambda)$ or $\bar{b}_{p}(\lambda)$ is used. Therefore, the $D_{2}(\lambda)$ of the published data ${ }^{3)}$ is used for the time being. In the band of $510 \sim 720 \mathrm{~nm}, \mathrm{~L}_{2}(\lambda)$ shown in Fig. 2 is used.

(3) $L_{1}(\lambda)$ and $L_{2}(\lambda)$ take a negative value in some wavelength so that the finally used $\dot{D}_{1}(\lambda)$ and $\dot{\mathrm{D}}_{2}(\lambda)$ are determined by adding a predetermined value thereto so as to make the lowest spectral optical density equal to zero. The values of $\dot{D}_{1}(\lambda)$ and $\dot{D}_{2}(\lambda)$ determined in such a way are shown in Table 3 .

Table 3 Improved estimated value of spectral optical densities of lens and macular pigment $\dot{D}_{1}(\lambda)$ and $\dot{D}_{2}(\lambda)$

\begin{tabular}{cccccc}
\hline $\begin{array}{c}\text { Wave- } \\
\text { length }\end{array}$ & $\dot{\mathrm{D}}_{1}$ & $\dot{\mathrm{D}}_{2}$ & $\begin{array}{c}\text { Wave- } \\
\text { length }\end{array}$ & $\dot{\mathrm{D}}_{1}$ & $\dot{\mathrm{D}}_{2}$ \\
\hline 390 & 1.680 & 0.074 & 560 & 0.080 & 0.183 \\
400 & 1.220 & 0.154 & 570 & 0.070 & 0.182 \\
410 & 0.840 & 0.229 & 580 & 0.060 & 0.159 \\
420 & 0.570 & 0.369 & 590 & 0.051 & 0.166 \\
430 & 0.390 & 0.434 & 600 & 0.044 & 0.153 \\
440 & 0.290 & 0.469 & 610 & 0.038 & 0.129 \\
450 & 0.245 & 0.529 & 620 & 0.032 & 0.104 \\
460 & 0.215 & 0.564 & 630 & 0.028 & 0.072 \\
470 & 0.195 & 0.514 & 640 & 0.024 & 0.071 \\
480 & 0.175 & 0.484 & 650 & 0.016 & 0.063 \\
490 & 0.160 & 0.479 & 660 & 0.007 & 0.049 \\
500 & 0.145 & 0.344 & 670 & 0.000 & 0.032 \\
510 & 0.130 & 0.250 & 680 & 0.001 & 0.020 \\
520 & 0.120 & 0.297 & 690 & 0.007 & 0.005 \\
530 & 0.110 & 0.181 & 700 & 0.009 & 0.000 \\
540 & 0.100 & 0.158 & 710 & 0.009 & 0.003 \\
550 & 0.090 & 0.184 & 720 & 0.009 & 0.009 \\
\hline
\end{tabular}

\section{Estimation of CMF's by Using $\dot{D}_{1}(\lambda)$ and $\dot{D}_{2}(\lambda)$}

In Paragraph 3, the improved estimated values of the spectral optical densities of lens and macular pigment $\dot{\mathrm{D}}_{1}(\lambda)$ and $\dot{\mathrm{D}}_{2}(\lambda)$ were determined. By using these, each CMF of Stiles' 20 observers will be estimated. The equation for estimation is given by

$$
\begin{aligned}
<\log \left[\overline{\mathrm{t}}_{\mathrm{p}}(\lambda) / \overline{\overline{\mathrm{t}}}(\lambda)\right]> & =\mathrm{k}_{1 \mathrm{p}}\left[\dot{\mathrm{D}}_{1}(\lambda)-\dot{\mathrm{D}}_{1}\left(\lambda_{\mathrm{t}}\right)\right] \\
& +\mathrm{k}_{2 \mathrm{p}}\left[\dot{\mathrm{D}}_{2}(\lambda)-\dot{\mathrm{D}}_{2}\left(\lambda_{\mathrm{t}}\right)\right],
\end{aligned}
$$

where $t$ represents $\mathrm{r}, \mathrm{g}$, or $\mathrm{b}$; bracket $<\quad>$ means that the value in $<\quad>$ is an estimated value; $k_{1} p$ and $k_{2 p}$ are coefficients showing the individual variations of the spectral optical densities of lens and macular pigment, respectively; and $\lambda_{\mathrm{r}}, \lambda_{\mathrm{g}}, \lambda_{\mathrm{b}}$ are the reference wavelengths of CMF's, wherein $\lambda_{\mathrm{I}}=645.2 \mathrm{~nm}, \lambda_{\mathrm{g}}=$ $526.3 \mathrm{~nm}, \lambda_{b}=444.4 \mathrm{~nm}$. Of course, the coefficients $k_{1 p}$ and $k_{2 p}$ are constant independently of CMF's.

The values of $\mathrm{k}_{1 \mathrm{p}}$ and $\mathrm{k}_{2 \mathrm{p}}$ were estimated by the least square calculation of the following value;

$$
\begin{aligned}
E= & \sum_{\lambda=390}^{720}\left\{\log \left[\overline{\mathrm{r}}_{\mathrm{p}}(\lambda) / \overline{\overline{\mathrm{r}}}(\lambda)\right]-<\log \left[\overline{\mathrm{r}}_{\mathrm{p}}(\lambda) /\right.\right. \\
& \overline{\overline{\mathrm{r}}}(\lambda)]>\}^{2} \\
& +\sum_{\lambda=390}^{550}\left\{\log \left[\mathrm{b}_{\mathrm{p}}(\lambda) / \overline{\mathrm{b}}_{\mathrm{p}}(\lambda)\right]-<\log \right. \\
& {\left.\left[\overline{\mathrm{b}}_{\mathrm{p}}(\lambda) / \overline{\overline{\mathrm{b}}}(\lambda)\right]>\right\}^{2}, }
\end{aligned}
$$

where $\log \left[\bar{r}_{p}(\lambda) / \overline{\bar{r}}(\lambda)\right]$ and $\log \left[\bar{b}_{p}(\lambda) / \overline{\bar{b}}(\lambda)\right]$ are based on the actual measured values.

In Eq. (8), $k_{1 p}$ and $k_{2 p}$ are estimated by using both CMF's of $\overline{\mathrm{r}}_{\mathrm{p}}(\lambda)$ and $\bar{b}_{\mathrm{p}}(\lambda)$ in the band below $550 \mathrm{~nm}$ and only $\overline{\mathrm{rb}}(\lambda)$ in the band above $560 \mathrm{~nm}$. The correlations between obtained $\mathrm{k}_{1} \mathrm{p}$ and $\mathrm{k}_{2 \mathrm{p}}$ and the principal component points $\mathrm{C}_{\mathrm{p}}{ }^{(1)}$ and $\mathrm{C}_{\mathrm{p}}{ }^{(2)}$ described in Paragraph 2 were high.

Now, in XYZ system, the deviations of each CMF's from the mean CMF's $\Delta \bar{x} p(\lambda), \Delta \bar{y} p(\lambda)$ $\Delta \bar{Z}_{p}(\lambda)$ will be discussed. The values in $X Y Z$ system are defined from the values in RGB system by the conversion formula ${ }^{7)}$ in CIE 1964 standard colorimetric system.

It can be understood that three types of values are obtainable for these deviations. That is to say that they are (1) the values obtained from actual measured CMF's, (2) the estimated values obtained from the published data of spectral optical densities $D_{1}(\lambda)$ and $D_{2}(\lambda)$ of 
lens and macular pigment respectively, as shown in the last report and (3) the estimated values obtained by using $\dot{\mathrm{D}}_{1}(\lambda)$ and $\dot{\mathrm{D}}_{2}(\lambda)$ of the present report.

By comparing these 3 types of CMF's deviations for all of Stiles' 20 observers, it was studied whether the improvement of approximation to the 1st deviations was obtained by the 3rd deviations better than by the 2nd deviations. The result is shown in Table 4.

Table 4 Comparison of improvement of 3 types of $C M F^{\prime}$ 's deviations

\begin{tabular}{cccc}
\hline CMF's deviation & Improved & Not improved & Worsoned \\
\hline$\triangle \overline{\mathrm{x}} \mathrm{p}(\lambda)$ & 11 & 4 & 5 \\
$\triangle \overline{\mathrm{y}} \mathrm{p}(\lambda)$ & 5 & 13 & 2 \\
$\triangle \overline{\mathrm{z}} \mathrm{p}(\lambda)$ & 1 & 18 & 1 \\
\hline
\end{tabular}

In Table $4, \Delta \bar{x}_{p}(\lambda), \Delta \bar{y}_{p}(\lambda), \Delta \bar{z}_{p}(\lambda)$ show the kinds of compared CMF's. Columns of "improved," "not improved" and "worsened" show the number of observers in whom the conformity of the estimated CMF's deviations to the actual measured CMF's deviations was "improved", "not improved" and "worsened" by being changed from $D_{1}(\lambda), D_{2}(\lambda)$ to $\dot{D}_{1}(\lambda)$, $\dot{D}_{2}(\lambda)$. It is obvious from Table 4 that an improvement does not be found for $\Delta \bar{y}_{p}(\lambda)$, $\Delta \bar{z}_{p}(\lambda)$ which have lower sensitivity in the long wavelength band. However, for $\Delta \bar{x}_{\mathrm{p}}(\lambda)$, the number of "improved", 11, is larger than the number of "worsened", 5 , so that it can be said that the change from $D_{1}(\lambda), D_{2}(\lambda)$ to $\dot{D}_{1}(\lambda)$, $\dot{\mathrm{D}}_{2}(\lambda)$ has improved the conformity of the estimated values of each CMF's to the actual measured CMF's for Stiles' 20 observers.

\section{Singular Value Decomposition of CMF's Deviations by Using $\dot{D}_{1}(\lambda), \dot{D}_{2}(\lambda)$}

In Paragraph 4, the CMF's deviations were obtained by using the improved estimated values $\dot{\mathrm{D}}_{1}(\lambda), \dot{\mathrm{D}}_{2}(\lambda)$ of the sprectral optical densities of lens and macular pigment. A singular value decomposition ${ }^{9)}$ is applied by summarizing these to a matrix as shown in Eq. (4) of the previous report ${ }^{8)}$.

By using singular value decomposition, the CMF's deviations can be composed as follows.
$\left[\begin{array}{cc}\Delta \bar{x}_{p}(\lambda) \\ \Delta \mathrm{y}_{p}(\lambda) \\ \Delta \mathrm{z}_{\mathrm{p}}(\lambda)\end{array}\right]=\sum_{\mathrm{i}=1}^{\mathrm{n}}\left\langle\mathrm{C}_{\mathrm{p}}{ }^{(\mathrm{i})}\right\rangle \cdot\left[\begin{array}{l}\left.<\alpha_{\mathrm{i}}(\lambda)\right\rangle \\ \left\langle\beta_{\mathrm{i}}(\lambda)\right\rangle \\ \left\langle\mathrm{r}_{\mathrm{i}}(\lambda)\right\rangle\end{array}\right] \ldots$.

The contribution factor of each component obtained by the singular value decomposition amounts to $83.3 \%$ and $16.1 \%$ in the 1st and 2nd component, respectively, and thereby the cumulative contribution factor is $99.4 \%$ which is so high that Eq. (9) is sufficient in $\mathrm{n}=2$. $\left\langle\alpha_{i}(\lambda)\right\rangle,\left\langle\beta_{i}(\lambda)\right\rangle,\left\langle\gamma_{i}(\lambda)\right\rangle(i=1,2)$ denote the estimated values of deviation functions for the 1 st and the 2 nd deviate observers. $\left\langle\mathrm{C}_{\mathrm{p}}{ }^{(\mathrm{i})}\right\rangle$ $(i=1,2)$ denotes the scores corresponding to the 1st and the 2nd component. By substituting these values for Eq. (9)., the CMF's deviations of Stiles' 20 observerss can be estimated.

Figs. 5 and 6 show a comparison of the deviation functions (solid line) obtained from the measured CMF's in the previous report with the deviation functions (dot line) obtained in this Paragraph. Figs. 5 and 6 correspond to the 1st and the 2nd deviation functions, respectively, and $\alpha(\lambda), \beta(\lambda), \gamma(\lambda)$ correspond to $X, Y, Z$ component, respectively.

From these figures, it is noticed that in the 1st deviation functions both lines conform to each other very well. On the other hand, in the 2nd deviation functions the conformity is not necessarily well due to the lower contribution factor. However, it is found that concerning the displacement of the actual measured deviation functions and the estimated deviation functions the 1st and the 2nd component displaces so as to compensate each other. At the wavelength of $600 \mathrm{~nm}$, for example, the estimated value $\left\langle\alpha_{1}(\lambda)\right\rangle$ displaces into the positive direction from the actual measured value $\alpha_{1}(\lambda)$ on one hand and the estimated value $\left\langle\alpha_{2}(\lambda)\right\rangle$ displaces into the negative direction from the actual measured value $\alpha_{2}(\lambda)$ on the other hand. That is to say that by adding $\alpha_{1}(\lambda)$ and $\alpha_{2}(\lambda)$ appropriately at the wavelength of $600 \mathrm{~nm}$ a conformity of the actual measurement and the estimation result can be expected. This characteristic can be also found at any wavelength where there exists a displacement of the actual measurement from the estimation at the other 

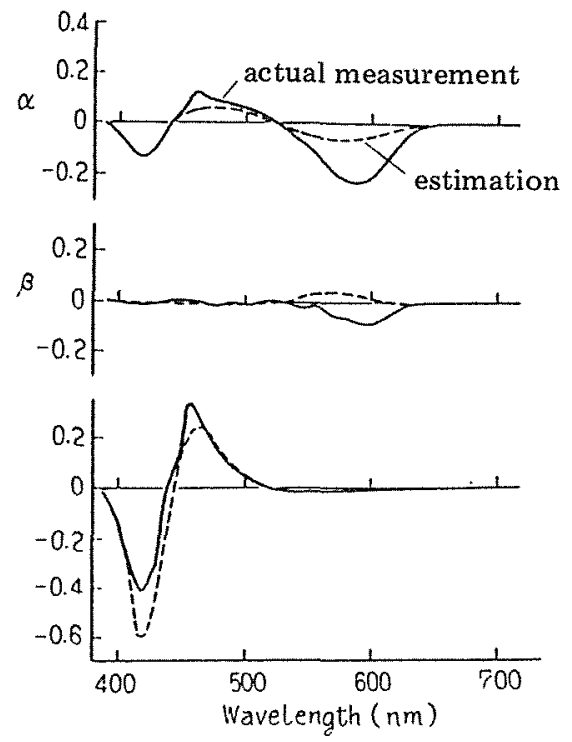

Fig. 5 Comparison of actual measured CMF's with estimated CMF's based on physiological information in the 1 st deviate observer

- The 1st deviate observer obtained from actual measured CMF's

... The 1st deviate observer obtained from estimated CMF's based on the improved estimated value $\dot{D}_{1}(\lambda)$, $\dot{D}_{2}(\lambda)$ of spectral optical densities of lens and macular pigment

wavelength of $\alpha(\lambda)$ and in $\beta(\lambda), \gamma(\lambda)$. This characteristic is very interesting because it shows an close relationship between the actual measured CMF's and the estimated CMF's.

\section{Discussion}

Pokomy et al. ${ }^{10)}$ estimated the age effect of lens optical density on the assumption that all of the individual variations of CMF's of Stiles and Burch depend on lens. Accoridng to them, the lens optical density $T_{L}(\lambda)$ for the average observer older than 20 is given by

$\mathrm{T}_{\mathrm{L}}(\lambda)=\mathrm{T}_{\mathrm{L}_{1}}(\lambda)[1+0.02(\mathrm{~A}-32)]+\mathrm{T}_{\mathrm{L}_{2}}(\lambda)$,

where $A$ is age; $T_{L 1}(\lambda)$ is a component affected by age; and $T_{L_{2}}(\lambda)$ is a constant component. $\mathrm{T}_{\mathrm{L} 1}(\lambda)$ corresponds to 1.33 times of the published data $D_{1}(\lambda)$ according to Wyszecki and Stiles.

According to Eq. (10), the density difference between the observers of 20 and 60 years old is $1.33 \mathrm{D}_{1}(\lambda) \times 0.8=1.064 \mathrm{D}_{1}(\lambda)$. On the other

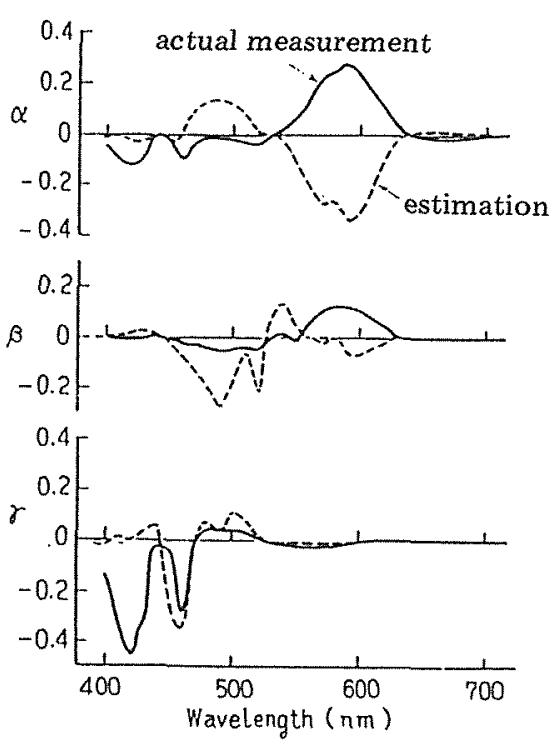

Fig. 6 Comparison of actual measured CMF with estimated CMF's based on physiological information in the 2nd deviate observer

- The 2nd deviate observer obtained from actual measured CMF's

... The 2nd deviate observer obtained from estimated CMF's based on the improved estimated value $\dot{D}_{1}(\lambda)$, $\dot{D}_{2}(\lambda)$ of spectral optical densities of optical densities of lens and macular pigment

hand, according to the principal component analysis of Paragraph 2, the contribution of lens to the spectral optical density variation is given by $C_{p}{ }^{(1)} L_{1}(\lambda)$, where $L_{1}(\lambda)$ is the 1 st eigen vector and $C_{p}{ }^{(1)}$ is the corresponding principal component score. As already described, $2.36 \times L_{1}(\lambda)$ corresponds to $D_{1}(\lambda)$ of the published data so that the above-mentioned contribution corresponds to $\mathrm{C}_{\mathrm{p}}{ }^{(1)} \mathrm{D}_{1}(\lambda) / 2.36$. In reference to Table 2 , the age of Stiles' 20 observers ranges from about 20 to about 60 and the range of $\mathrm{Cp}^{(1)}$ is $1.123+1.823=2.946$. Therefore, the density difference between the observers of 20 and 60 years old is estimated as $1.248 \mathrm{D}_{1}(\lambda)$. Although Pokorny et al. and the authers used a respective different analysis, the values obtained in both analyses for the lens optical density difference show good conformity.

Concerning macular pigment, our results show that the 2nd principal component score ranges from -0.359 to 0.502 (see Table 2) and the peak value of the 2 nd eigen vector at peak wave- 
length is 0.491 (see Table 1). The macular pigment optical density range estimated from these values becomes $(0.502+0.359) \times 0.491=0.423$. Also, for Stiles' observers, as shown by Ichikawa ${ }^{4)}$, if zero density value is assigned to the observer with the lowest macular pigment density, the individual density of the observer with the highest macular pigment density becomes 0.423 at the peak wavelength. The value of mean density is lower than that and may be estimated as about a half. This result is obviously lower than the measured values already reported concerning macular pigment. In $\mathrm{D}_{2}(\lambda)$ of the published data by Wyszecki and Stiles, the density value of 0.495 is given at the peak wavelength. In the recent measured values by Pease et al. ${ }^{11)}$ the optical density of 0.77 was obtained at the peak wavelength.

The lower optical density estimated from the principal component analysis can be explained as follows. The data used for the principal component analysis of the present report are the measured values of CMF's at $10^{\circ}$ visual field by Stiles. This result may be considered as the mean value of CMF's at the retina surface within $5^{\circ}$ radius around fovea centralis. By the way, it is reported $^{12)}$ that the macular pigment is found in a maximum amount at the fovea centralis and its content decreases towards the periphery, resulting in that it is not almost found on the retina position far from the fovea centralis by $4^{\circ}$ or more. The contribution from macular pigment included in Stiles' CMF's is considered as the mean of the contribution from macular pigment within $10^{\circ}$ visual field. This mean optical density must be smaller than the optical density at the fovea centralis. Thereby, it is supposed that the estimated value from the CMF's analysis is smaller than the measured value at the fovea centralis.

\section{Conclusion}

The results obtained in the present report may be summarized as follows.

(1) From the analysis on the blue and the red CMF's of Stiles' 20 observers, improved estimated values of the spectral optical densities of lens and macular pigment were obtained.

(2) The published data of lens spectral optical density showed a good conformity to the estimated values based on the CMF's by the present report. Concerning macular pigment spectral optical density, in the wavelength band below $450 \mathrm{~nm}$ the estimated values showed a little higher values than the published data. On the other hand, in the wavelength band above $500 \mathrm{~nm}$ the spectral optical densities of the estimated values were not constant but showed a systematic declination along with the increase of wavelength.

(3) Based on the previous report ${ }^{1)}$ and the analysis result of the present report, it was found that the individual variations of the CMF's of the normal observers could be explained by the individual variations of the spectral optical densities of lens and macular pigment.

(4) By using the improved estimated values of the spectral optical densities of lens and macular pigment (Tables 1 and 2), the improved estimated values can be given to the actual measured CMF's of Stiles 20 observers compared to the previous report ${ }^{1)}$.

(5) By applyint the singular value decomposition to the CMF's deviate functions of Stiles' 20 observers estimated based on the improved estimated values of the spectral optical densities of lens and macular pigment, a result similar to and closely related to the deviate observers in the previous report could be obtained.

\section{Acknowledgement}

The auther wishes to thank Mr. Yoshio Matsumoto (4th (1986) graduate of Management Eng. Dept. of Osaka Electro-Communication University) for the cooperation of performing an enormous mathematical analysis and preparing a number of figures and tables.

\section{References}

(1) Nayatani, Y., Takahama, K. and Sabagaki, H.: Physiological Causes of Individual Variations of Color Maching Functions, Color Res. Appl. 13 (1988) 289-297.

(2) Stiles, W.S. and Wyszecki, G.: Color Science, 2nd ed., Wiley, New York, (1982) pp. 817-821

(3) Stiles, W.S. and Wyszecki, G.: Color Science, 2nd ed., Wiley, New York (1982) pp. 107-116, $\mathrm{D}_{1}(\lambda)$ : Table $1(2,4,6)$ of p. $109 ; \mathrm{D}_{2}(\lambda)$ : Table $2(2,4,6)$ of p. 112

(4) Ichikawa, H.: Aging and Function of Eye, 
Clinical Ophthalmology Vol. 35 (1981) pp. 9-26

(5) Naylor, E.J. and Stanworth, A.: Retinal Pigment and the Haidinger Effect, J. Physiol. (London), 124 (1954) 543

(6) Stiles, W.S.: Further studies of visual mechanisms by the two-color threshold method, Coloq. Probl. Opt. Vis. (U.I.P.A.P., Madrid) 1 (1953) 65-103

(7) Stiles, W.S. and Wyszicki, G.: Color Science, 2nd ed., Wiley, New York, Equation [8 (3.3.3)] (1982) p. 141

(8) Nayatani, Y., Takahama, K. and Sobagaki, H.: A Proposal of New Standard Deviate Observers, Color Res. \& Appl. 8 (1983) pp. 47-56

(9) Mori, T., Natori, R.and Torii, T.: Numerical
Image Calculation, Chapt. 3, Iwanami Book (1982)

(10) Pskorny, J., Smith, V.C. and Lutze, M.: Aging of the Human Lens, Appl. Opt. 26 (1987) 1437-1440

(11) Pease, P.L., Adams, A.J. and Nuccio, E.: Optical Density of Human Macular Pigment, Vision Res. 27 (1987) 705-710

(12) Viénot, F.: Can Variation in Macular Pigment Account for the Variation of Colour Matches with Retinal Position?. In: Mollon, J.D. and Sharpe, L.T.: Colour Vision, Academic Press, (1983) pp. $107-116$

Received January 28, 1988: Accepted May 14. 